\title{
Confinement-induced columnar crystals of ellipses
}

\author{
Weiwei Jin (金炜炜)๑, Yuqian Wang (王昱乾)๑, Ho-Kei Chan (陈浩基)๑, ${ }^{*}$ and Zheng Zhong (仲政) \\ School of Science, Harbin Institute of Technology (Shenzhen), Shenzhen 518055, China
}

(Received 2 September 2020; accepted 21 December 2020; published 19 January 2021)

\begin{abstract}
Densest-packed structures of identical ellipses within a parallel strip have been obtained through Monte Carlo simulations and analytic derivations. Each of such structures is an affine transformation of a particular densest-packed structure of identical circular disks. Three types of structures have been identified: Types $\mathcal{X}$ and $\mathcal{Y}$ correspond, respectively, to affine stretches along the perpendicular and axial directions of the parallel strip, and type $\mathcal{M}$ corresponds to affine stretches of locally densest structures. Our findings enrich the field of condensed matter physics with a class of confinement-induced, orientationally ordered crystal structures, and offer possibilities of developing low-dimensional materials with specific orientational ordering.
\end{abstract}

DOI: 10.1103/PhysRevResearch.3.013053

\section{INTRODUCTION}

Packings of hard particles can serve as theoretical models for the ordered or disordered structures of a surprising variety of condensed matter systems: Examples for bulk systems include the structures of solids [1,2], liquids [3,4], glasses [2,5], and heterogeneous materials [6]. For self-assembled monolayers, such as those of colloids [7-12], nanoplates [13-17], octapods [18-20], and fullerenes [21], the corresponding twodimensional structures can be modeled as dense-packed layers of hard particles that mimic the shapes of the underlying atomic or molecular constituents. For columnar crystals that arise from the confinement of spherical-like constituents inside a cylindrical pore or tube, such as the columnar crystals of fullerenes [22,23], nanoparticles [24-26], hollow carbon spheres [27], colloids [28-30], microspheres [31], microbubbles [32], and polymeric particles [33], the corresponding quasi-one-dimensional structures may be represented theoretically by the densest-packed structures of identical spheres in cylindrical confinement [34-48].

In the past two decades, a variety of studies on the dense or densest ordered packings of shape-anisotropic hard particles have been carried out: Examples include the bulk ordered packings of ellipsoids [2,49], Platonic and Archimedean solids [50], polyhedra [51], cube-octahedron interpolated structures [52], and spherotetrahedra [53], for which a variety of novel crystal structures have been discovered. Recent research [54] revealed that a combination of particle-shape anisotropy with geometric confinement could result in an unexpected variety of densest structures with unconventional translational or orientational order. For identical spheroids in

\footnotetext{
*epkeiyeah@yahoo.com.hk

Published by the American Physical Society under the terms of the Creative Commons Attribution 4.0 International license. Further distribution of this work must maintain attribution to the author(s) and the published article's title, journal citation, and DOI.
}

cylindrical confinement [54], such complex structures arise as a result of the competition between confinement-induced chiral ordering and shape-anisotropy-induced orientational ordering. For two-dimensional systems, there has been a comprehensive study [55] on the densest-packed structures of identical circular disks within a parallel strip, but to our surprise there has not been any extension of this study to ellipses, which are regarded as the simplest extensions from circular disks to include shape anisotropy, despite active research on the packings of ellipses in many other contexts [56-68].

In this paper, we report a class of confinement-induced, orientationally ordered columnar crystals as obtained from the densest possible packings of identical hard ellipses within an infinitely long parallel strip. Such structures, which exhibit periodicity along the axial $y$ direction of the confining strip, are hereafter referred to as columnar crystals of ellipses. For particles in unbounded two-dimensional space, the densest-packed structure of identical ellipses of any aspect ratio $A \geqslant 1$ is related to the densest-packed hexagonal structure of identical circular disks via an affine transformation [56], constituting an infinite set of crystal structures with the same packing fraction of $\phi=\pi / \sqrt{12} \approx 0.9069$. Our numerical simulations revealed that any densest-packed structure of identical ellipses in strip confinement is also an affine transformation (Fig. 1) of a particular densest-packed structure of circular disks as reported in Ref. [55]. Based on this general result, we propose an analytic theory that describes all such strip-confined densest-packed structures of ellipses in a unified framework, where we classify such structures into three types and give a full account of all possible structural transitions from one type to another.

This paper is organized as follows: In Sec. II, we review the solutions to a related problem of disk packing [55], where these solutions form the basis of our subsequent discussion and analysis of ellipse packings. In Sec. III, we discuss our method of simulation, and present our numerical findings on the dense packings of ellipses. In particular, we show that all the particles in a densest-packed structure are necessarily aligned along the same direction for a maximization of the 
(a)

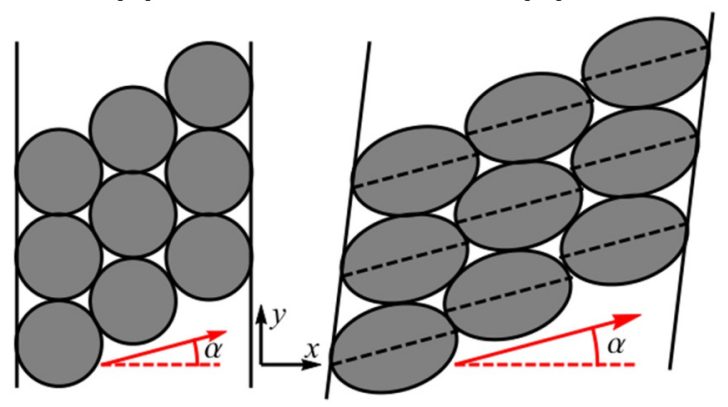

FIG. 1. An affine stretch of the locally densest type- $\mathcal{M}$ structure of circular disks at $D=D_{\min }$ of $n=3$, by a factor of $f_{\mathrm{st}}=1.5$ along the direction of $\alpha=\pi / 12$. Each disk in (a) is transformed into an ellipse in (b) with an aspect ratio of $A=f_{\text {st }}=1.5$. The long axis of each resulting ellipse is aligned along the stretch direction.

packing fraction $\phi$. In Sec. IV, we present a comprehensive analytic theory for all such densest-packed structures of ellipses, based on the general result that every densest-packed structure of ellipses is an affine transformation of a particular densest-packed structure of circular disks. In particular, we discuss three types of densest-packed structures of ellipses and the nature of the corresponding structural transitions from one type to another. In Sec. V, we discuss the significance of our findings, some potential applications of such confinementinduced crystal structures of ellipses, as well as some possible extensions of this research.

\section{ANALYTIC SOLUTIONS TO DENSEST PACKINGS OF CIRCULAR DISKS}

The densest-packed structures of identical circular disks $(A=1)$ within a parallel strip [55] form the basis of our subsequent discussion of ellipse packings, and therefore we begin with a brief review of such structures. At any value of $D$, the corresponding densest-packed structure of circular disks is generally a zigzaglike arrangement of equal-sized equilateraltriangular-shaped clusters of close-packed disks (Fig. 2). Each cluster, which generally consists of $n(n+1) / 2$ particles for a center-to-center side length of $(n-1) b$, has one of its edges adhered to either boundary, with each boundary sharing half of the clusters in an alternating fashion. For the specific case of $n=1$, such a triangular-shaped cluster is essentially a single particle with a zero center-to-center side length, but the following general solutions for densest packings of circular disks apply to this specific case as well. For densest-packed structures of ellipses, where such structures are all affine transformations of particular densest-packed structures of circular disks, the corresponding general solutions also apply to the specific case of $n=1$.

Any adjacent pair of clusters generally forms a rhombiclike unit cell with $n(n+1)$ particles, where, for the specific case of $n=1$, this unit cell is just a pair of circular disks in mutual contact. For such a generally rhombiclike unit cell, the corresponding size ratio $D$ must be within the interval of

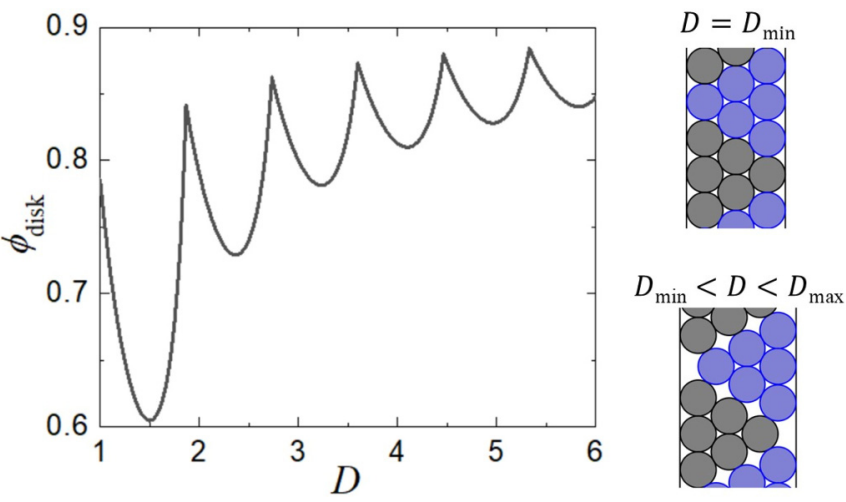

FIG. 2. Plot of the packing fraction $\phi_{\text {disk }}$ as a function of $D$ for densest-packed structures of identical circular disks in strip confinement [55]. Two such structures at $n=3$ are displayed on the right-hand side of the figure. The primitive unit cell of the locally densest type- $\mathcal{M}$ structure at $D=D_{\min }$ consists of only $n=3$ particles.

$\left[D_{\min }, D_{\max }\right)$, where

$$
\left\{\begin{array}{l}
D_{\min }=1+\frac{\sqrt{3}}{2}(n-1) \\
D_{\max }=1+\frac{\sqrt{3}}{2} n
\end{array} .\right.
$$

For any value of $n \geqslant 1$, this interval of $D$ takes on a constant width of $\sqrt{3} / 2$, with the upper bound $D_{\max }$ of $n$ being equal to the lower bound $D_{\min }$ of $(n+1)$.

For the densest-packed structure at any value of $D$, the packing fraction is given by

$$
\phi_{\text {disk }}=\frac{n(n+1) \pi}{4 D\left\{n-1+\sqrt{4-[\sqrt{3}(n-1)-2(D-1)]^{2}}\right\}}
$$

and is displayed in Fig. 2. It exhibits a local maximum at $D=$ $D_{\min }$, and reaches a local minimum at $D=D_{\min }+1 / 2$. At $D=D_{\min }$, the locally densest structure, which we refer to as a type- $\mathcal{M}$ structure of circular disks, is an extraction from the densest-packed hexagonal structure of identical circular disks in unbounded two-dimensional space. Its primitive unit cell is made up of only $n$ particles, which is different from the general case of a rhombiclike unit cell, and its packing fraction is given by

$$
\phi_{\mathcal{M}}=\frac{n \pi}{4+2 \sqrt{3}(n-1)}
$$

according to Eq. (2).

\section{COMPUTATIONAL INVESTIGATIONS OF DENSEST PACKINGS OF ELLIPSES}

\section{A. Method of simulation}

For $a \geqslant b$, let $a$ and $b$ be, respectively, the long and short axes of an ellipse within a parallel strip of width $d$, and $A \equiv a / b$ be the corresponding aspect ratio. For given values of the strip-to-ellipse size ratio $D \equiv d / b$, densest-packed structures of identical ellipses were obtained via a Monte Carlo approach [49,50], where this approach is known to be efficient for generating optimal structures of anisotropic particles. Simulations were carried out within a parallelogram-shaped cell, 


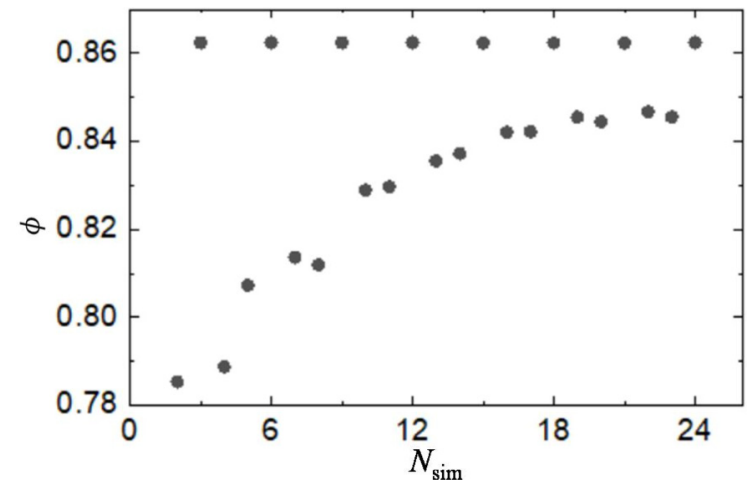

FIG. 3. Plot of the packing fraction $\phi$ as a function of the number of particles, $N_{\text {sim }}$, in the simulation cell, for dense-packed structures of identical ellipses at $A=1.1$ and $D=3$. If $N_{\text {sim }}$ is a multiple of the number of particles, $N_{\mathrm{UC}}=3$, in the unit cell, the packing fraction $\phi$ attains its maximum value and the corresponding densest-packed structure is obtained.

with the pair of edges along the $y$ direction being the parallel boundaries of the strip and the other pair of edges being the periodic boundaries of each simulation. For $A \in(1,10]$ and $D \in[1,10]$, close-packed structures with, respectively, $N_{\text {sim }}=1$ to 110 ellipses were generated, with each structure obtained via the slow compression of a random packing with an initial packing fraction of 0.1. As illustrated in Fig. 3, the packing fraction $\phi$ attains its maximum value if the number of particles, $N_{\text {sim }}$, in the simulation cell is a multiple of the number of particles, $N_{\mathrm{UC}}$, in the unit cell of the corresponding simulated structure.

\section{B. Excess volume from grain boundaries}

Our Monte Carlo simulations revealed that any densestpacked structure of identical ellipses in strip confinement is an affine transformation of a particular densest-packed structure of identical circular disks. All the particles in such a densestpacked structure are aligned along the same direction, and any dense-packed structure with multiple orientations of particles is not a candidate for densest-packed arrangements. This general result can be understood in terms of the excess-volume effects of grain boundaries between adjacent crystalline regions in a polycrystalline system [69,70]: As illustrated in Fig. 4, the packing density of a bidirectional dense-packed crystal structure with two possible orientations of particles is generally lowered by the presence of grain boundaries between adjacent regions of different particle orientations. The packing density $\phi$ generally decreases as the number of grain boundaries, $N_{\mathrm{GB}}$, increases, where the highest value of $\phi$ at $N_{\mathrm{GB}}=0$ corresponds to a specific case of uniform particle orientations in the complete absence of grain boundaries.

\section{Excess volume from nonuniform orientations of particles}

The effects of any nonuniformity in particle orientations on the packing density $\phi$ can be studied in a more general context by considering a two-dimensional nematic-order parameter $S$ [71]. This order parameter is the positive eigenvalue of the (a)
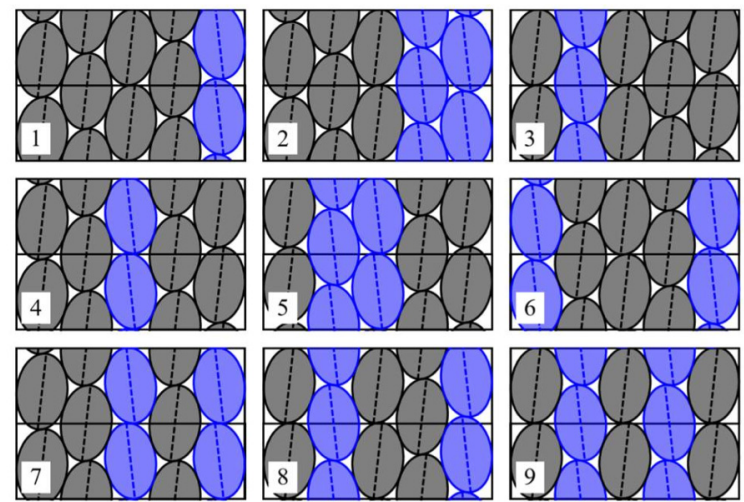

(b)

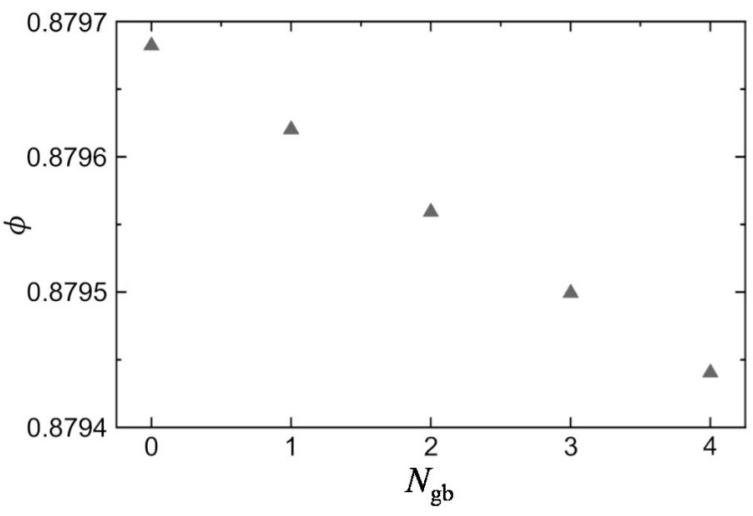

FIG. 4. Bidirectional dense-packed crystal structures of identical ellipses in strip confinement at $A=1.5$ and $D=4.5$, with a total of $N_{\mathrm{UC}}=5$ particles in the unit cell of each structure: (a) Illustration of nine bidirectional structures. There is the presence of a grain boundary between adjacent regions of different particle orientations in each of structures 1 and 2, two such grain boundaries in each of structures 3 to 6 , three such grain boundaries in each of structures 7 and 8, and four such grain boundaries in structure 9. (b) Plot of the packing fraction $\phi$ as a function of the number of grain boundaries, $N_{\mathrm{GB}}$. The packing fraction $\phi$ decreases in a linearlike fashion for increasing $N_{\mathrm{GB}}$. The highest value of $\phi$ at $N_{\mathrm{GB}}=0$ corresponds to a specific case of uniform particle orientations in the complete absence of grain boundaries.

order tensor

$$
Q=\frac{1}{N_{\mathrm{UC}}} \sum_{i=1}^{N_{\mathrm{UC}}}\left(2 \hat{u}_{i} \otimes \hat{u}_{i}-I\right),
$$

where $\hat{u}_{i}$ is the unit vector specifying the orientation of ellipse $i$, and $I$ is the identity matrix. Any case of a strictly uniform alignment of particles corresponds to the limiting value of $S=1$ for perfect nematic ordering, and any nonuniformity in particle orientations corresponds to a less-than-unity value of $S$. For dense-packed structures obtained from our Monte Carlo simulations, the value of $\phi$ in any case of $S<1$ is smaller than the maximum value of $\phi$ at $S=1$, as illustrated in Fig. 5 for cases with $N_{\mathrm{UC}}=24$ particles in the unit cell of each structure. It is evident from these numerical results that all the particles in a densest-packed structure have to be aligned along the same direction for $\phi$ to be maximized. 


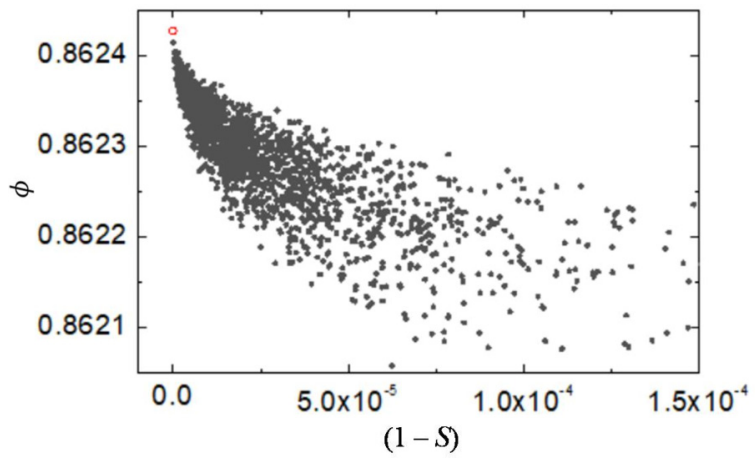

FIG. 5. Plot of the packing fraction $\phi$ as a function of $(1-S)$ for dense-packed structures at $A=1.1$ and $D=3$, where $S$ is a two-dimensional nematic-order parameter [71]. There are $N_{\mathrm{UC}}=24$ particles in the unit cell of each structure. The maximum value of $\phi$, as indicated by the red circle, corresponds to a scenario of perfect nematic ordering at $1-S=0$ where all the particles are aligned along the same direction.

\section{ANALYTIC THEORY FOR DENSEST PACKINGS OF ELLIPSES}

The general result, that every densest-packed structure of ellipses is an affine transformation of a particular densestpacked structure of circular disks, allows for a unified theoretical description of all such densest-packed structures of ellipses. Each of such structures is thought of as the result of an affine stretch along a particular unit vector $\hat{n}$, with the stretch factor $f_{\mathrm{st}} \geqslant 1$ being conveniently equal to the aspect ratio $A \geqslant 1$ of ellipses.

\section{A. Mathematical description of an affine stretch}

For any arbitrary vector $\vec{m}$, such an affine stretch is mathematically given by

$$
\overrightarrow{m^{\prime}} \equiv \vec{m}+(A-1)(\vec{m} \cdot \hat{n}) \hat{n},
$$

where $\vec{m}^{\prime}$ is the resulting vector after the transformation. Figure 1 illustrates an affine stretch of a particular type- $\mathcal{M}$ structure of circular disks. Each circular disk becomes an ellipse with its long axis aligned along the stretch direction. The area of each particle, and the total area of the parallel strip, are both multiplied by $A$, so that the packing fraction remains unchanged. The strip-to-ellipse size ratio $D \equiv d / b$ after the affine stretch is given by

$$
D=\frac{D_{0} A}{\sqrt{1+\left(A^{2}-1\right) \sin ^{2} \alpha}},
$$

where $D_{0}$ is the initial value of $D$, and $\alpha \in[0, \pi / 2]$ is the angle subtended between the stretch direction and the $x$ axis. For any chosen value of $A$, the value of $D$ increases monotonically for decreasing $\alpha$, from $D=D_{0}$ at $\alpha=\pi / 2$ for a stretch along the $y$ direction to $D=D_{0} A$ at $\alpha=0$ for a stretch along the $x$ direction.

\section{B. Three types of densest-packed structures}

Taking into account all possible values of $\alpha \in[0, \pi / 2]$, each data point in the plot of Fig. 2 should be replaced by a fi- (a)

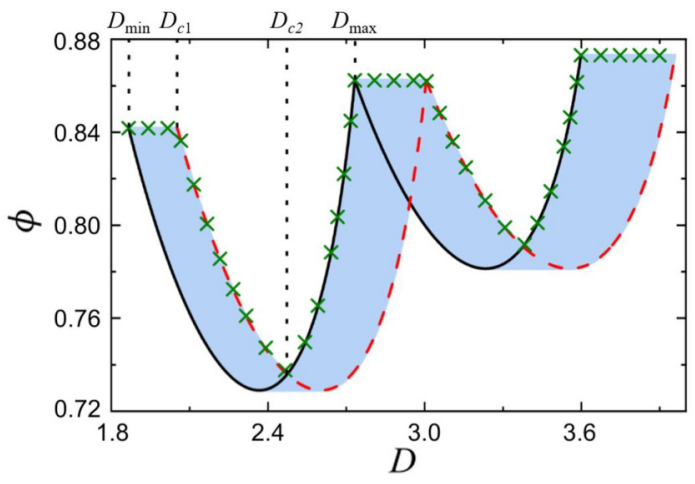

(b)

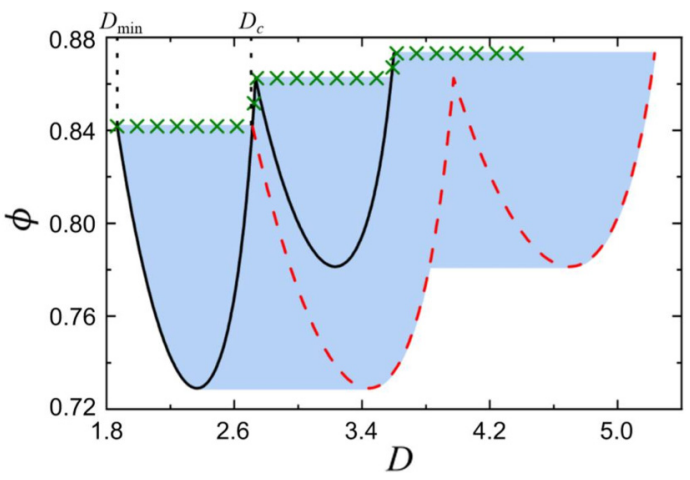

FIG. 6. Plots of the packing fraction $\phi$ as a function of $D$ for (a) $A=1.1$ and (b) $A=A_{c} \approx 1.454$. For each plot, the ranges $D \in$ $[1+\sqrt{3} / 2,1+\sqrt{3})$ and $[1+\sqrt{3}, 1+3 \sqrt{3} / 2)$ correspond, respectively, to the cases of $n=2$ and 3 ; the blue region represents the set of structures that are obtained from all possible values of $\alpha \in[0, \pi / 2]$; and the green crosses correspond to our numerical results of densest possible structures. The difference $\delta D \equiv D_{\max }-D_{c}$ is sufficiently small such that $D_{c}$ can be taken as a perturbation from $D_{\max }$.

nite span of $D$ from $D=D_{0}$ for $\alpha=\pi / 2$ to $D=D_{0} A$ for $\alpha=$ 0 . This is illustrated in Figs. 6(a) and 6(b) for, respectively, two different values of $A$. In each of these subfigures, the blue region represents the set of structures that are obtained from all possible values of $\alpha \in[0, \pi / 2]$, and the red dashed curve for $D=D_{0} A$ is obtained through a replacement of the variable $D$ in Eq. (2) by $D / A$. The densest-packed structures of ellipses correspond to the upper bound of the blue region. As illustrated in Fig. 6, a complete agreement between this theoretical upper bound and our numerical results of densestpacked structures (as indicated by the green crosses in the figure) has been obtained. For $n=2$ and 3, Fig. 6(a) displays a scenario in which the interval of $D \in\left[D_{\min }, D_{\max }\right)$ for each value of $n$ is partitioned into three regimes that correspond, respectively, to three different types of densest-packed structures.

(1) For $D \in\left[D_{\min }, D_{c 1}\right]$ where $D_{c 1}=A D_{\min }$, each densestpacked structure of ellipses corresponds to an affine stretch of the locally densest type- $\mathcal{M}$ structure of circular disks at $D_{\min }$, with a unique stretch direction for each particular value of $D$. The packing fraction, which is given by Eq. (3) for the type- $\mathcal{M}$ structure of circular disks, does not vary with $D$ nor $A$, and it is a function of $n$ only. The resulting densest-packed structure at any values of $D$ and $A$ is hereafter referred to as a type- $\mathcal{M}$ structure of ellipses [Fig. 1(b)]. 

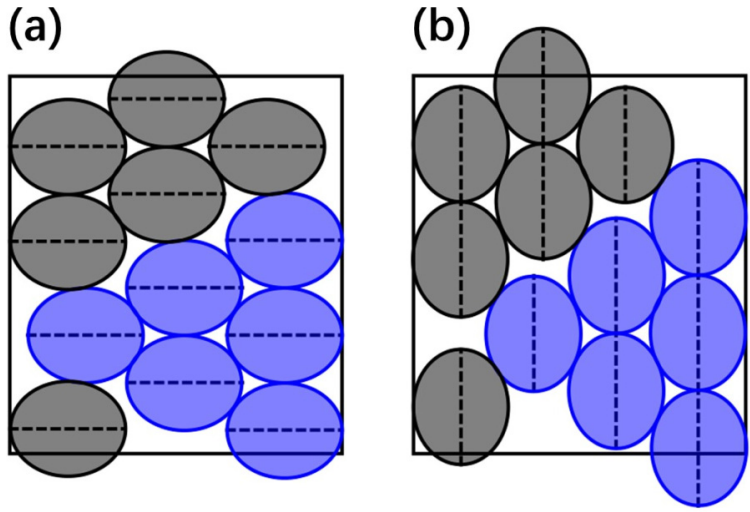

FIG. 7. Discontinuous transition at $n=3, D=3.5$, and $A \approx$ 1.212 between (a) a type- $\mathcal{X}$ structure $(\alpha=0)$ and (b) a type- $\mathcal{Y}$ structure $(\alpha=\pi / 2)$. In this structural transition, the two structures take on different values of $\alpha$ but share the same value of $\phi$.

(2) For $D \in\left[D_{c 1}, D_{c 2}\right]$, each densest-packed structure of ellipses, as indicated by the red dashed curve, corresponds to an affine stretch of a particular densest-packed structure of circular disks along the $x$ direction. We refer to such a densestpacked structure as a type- $\mathcal{X}$ structure of ellipses [Fig. 7(a)].

(3) For $D \in\left[D_{c 2}, D_{\max }\right)$, each densest-packed structure, which corresponds to an affine stretch along the $y$ direction, is referred to as a type- $\mathcal{Y}$ structure of ellipses [Fig. 7(b)]. The packing fraction $\phi$ of such a structure is independent of $A$, because an affine stretch of any value of $A$ along the $y$ direction will leave the values of both $D$ and $\phi$ unchanged, i.e., the same as those of the original structure of circular disks.

\section{Critical values of $A$ and $D$}

The three-regime scenario presented in Fig. 6(a) is valid only if $A$ is smaller than a critical parameter $A_{c}$. At $A=A_{c}$, we have $D_{c 1}=D_{c 2}=D_{c}=A_{c} D_{\min }$. As illustrated in Fig. 6(b), for $A \geqslant A_{c}$, the interval of $D \in\left[D_{\min }, D_{\max }\right)$ is only partitioned into two regimes, with each densest-packed structure at $D \in\left[D_{\min }, D_{c}\right]$ and $D \in\left[D_{c}, D_{\max }\right)$ being a type- $\mathcal{M}$ and a type- $\mathcal{Y}$ structure, respectively. Using Eqs. (1), (2), and (3), accurate values of $D_{c}$ and $A_{c}=D_{c} / D_{\min }$ for any chosen value of $n$ can be computed from the exact analytic solution of

$$
\phi_{\text {disk }}=\phi_{\mathcal{M}} .
$$

This solution is too lengthy to be presented here. Fortunately, by assuming $D_{c}$ to be a perturbation from $D_{\max }$, a first-order approximation that provides insights into the dependence of $D_{c}$ and $A_{c}$ on $n$ can be obtained:

$$
\delta D \equiv D_{\max }-D_{c} \approx \frac{2-\sqrt{3}}{4(n+\sqrt{3})}
$$

and

$$
A_{c}-1 \approx \frac{2 \sqrt{3} n+4+\sqrt{3}}{2\left[\sqrt{3} n^{2}+(5-\sqrt{3}) n+2 \sqrt{3}-3\right]} .
$$

For $n \rightarrow \infty$, the value of $D_{c}$ approaches that of $D_{\max }$ and the value of $A_{c}$ approaches unity.
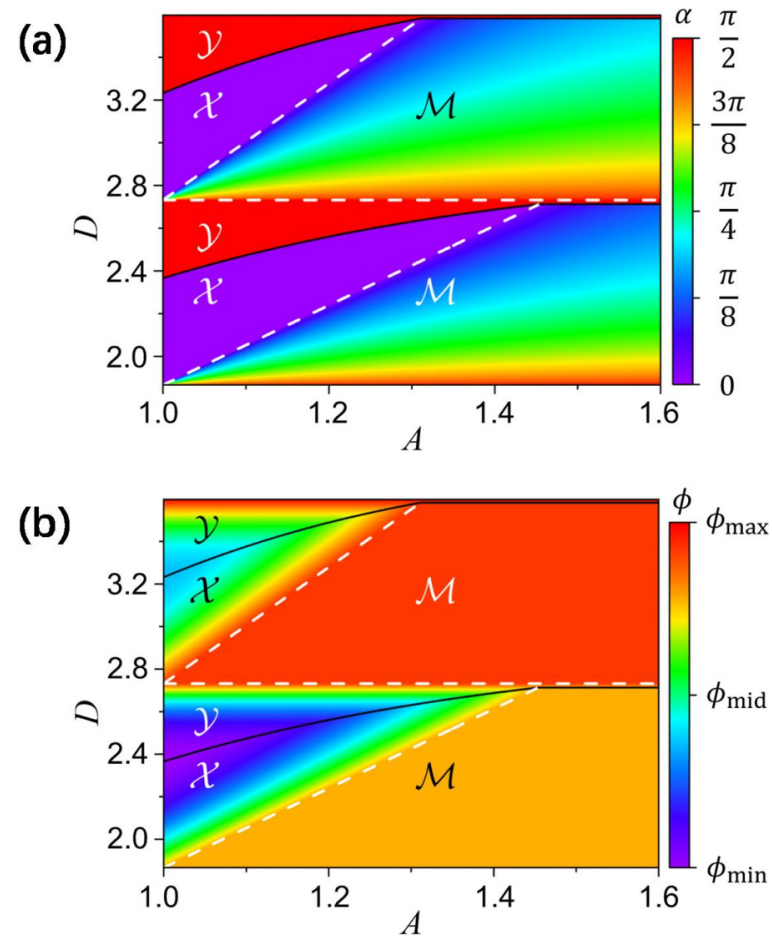

FIG. 8. Maps of densest-packed structures of ellipses for cases of $n=2$ and 3 at $D \in[1+\sqrt{3} / 2,1+\sqrt{3})$ and $[1+\sqrt{3}, 1+3 \sqrt{3} / 2)$, respectively. For each value of $n$, contour plots of the angle $\alpha$ and the packing fraction $\phi$ are presented in (a) and (b), respectively, with $\phi_{\max }=(2 \pi) /(2+3 \sqrt{3}) \approx 0.873, \phi_{\min }=(3 \pi) /[2(3+$ $2 \sqrt{3})] \approx 0.729$, and $\phi_{\operatorname{mid}} \equiv\left(\phi_{\max }+\phi_{\min }\right) / 2$ for the color scale of (b). Each of these maps consists of three distinct regions that correspond, respectively, to the three types of densest-packed structures $\mathcal{M}, \mathcal{X}$, and $\mathcal{Y}$. Interstructural boundaries for continuous transitions are indicated by white dashed curves, and those for discontinuous transitions are indicated by black solid curves.

\section{Maps of densest-packed structures}

For any chosen value of $n$, a theoretical map of densestpacked structures with $D \geqslant 1$ and $A>1$ as independent parameters can be constructed, as illustrated in Fig. 8 for the cases of $n=2$ and 3 . Here, we do not consider the limiting case of $A=1$ for circular disks, because the three types of densest-packed structures, $\mathcal{X}, \mathcal{Y}$, and $\mathcal{M}$, and the corresponding transitions from one type of structures to another, are only defined for ellipses at $A>1$ where the particles exhibit well-defined orientations. Such a map, which can be a contour plot of $\alpha$ [Fig. 8(a)] or a contour plot of $\phi$ [Fig. 8(b)], generally consists of three distinct regions that correspond, respectively, to the three types of densest-packed structures described above: The densest-packed structures at $D \in\left[D_{\min }, D_{c 1}\right]$ and $A \in\left(1, A_{c}\right)$, as well as those at $D \in\left[D_{\min }, D_{c}\right]$ and $A \geqslant A_{c}$, are all type- $\mathcal{M}$ structures, with each of them taking on a unique value of $\alpha$ as follows according to Eq. (6):

$$
\alpha_{\mathcal{M}}=\sin ^{-1} \sqrt{\frac{\left(\frac{D_{\min } A}{D}\right)^{2}-1}{A^{2}-1}} \in[0, \pi / 2] .
$$

At $D \in\left[D_{c 1}, D_{c 2}\right]$ and $A \in\left(1, A_{c}\right)$, the densest-packed structures are all type- $\mathcal{X}$ structures (i.e., $\alpha=0$ ). The rest are 
all type- $\mathcal{Y}$ structures (i.e., $\alpha=\pi / 2$ ). The boundary between one region and another is here referred to as an interstructural boundary. Within the map of any given value of $n$, there generally exist three interstructural boundaries, of types $\mathcal{M}-\mathcal{X}$, $\mathcal{M}-\mathcal{Y}$, and $\mathcal{X}-\mathcal{Y}$, respectively, as well as a "triple point" at $A=A_{c}$ and $D=D_{c}=A_{c} D_{\min }$ connecting all the three regions. The map also shares a boundary of type $\mathcal{M}-\mathcal{Y}$ with each of its neighboring maps, where these neighbors correspond to the cases of $(n+1)$ and $(n-1)$, respectively. As illustrated in Fig. 8, maps of different values of $n$ are similar to each other, where the map of $(n+1)$ appears to be a compression of the map of $n$ along the axis of $A$. At $n \rightarrow \infty$, the regime of $A<A_{c}$ and $D \in\left[D_{\min }, D_{\max }\right)$ as well as that of $A \geqslant A_{c}$ and $D \in\left[D_{c}, D_{\max }\right)$ vanish, such that any densest-packed structure across the map must be a type- $\mathcal{M}$ structure, as expected for the bulk limit of $D \rightarrow \infty$ [56].

\section{E. Nature of structural transitions}

At any interstructural boundary, the corresponding structural transition from one type of structure to another can be described as either continuous or discontinuous. In any continuous transition, the two structures involved become identical at the transition point, sharing the same values of the angle $\alpha$ and the packing fraction $\phi$. In any discontinuous transition, the two structures involved remain structurally different at the transition point. They take on different values of $\alpha$ but share the same value of $\phi$. It follows from these definitions that any structural transition must be accompanied by a continuity in $\phi$.

As shown in Fig. 8(a), for any value of $n$, any $\mathcal{M}-\mathcal{X}$ transition is continuous (white dashed curves) while any $\mathcal{M}-\mathcal{Y}$ or $\mathcal{X}-\mathcal{Y}$ transition is discontinuous (black solid curves), and any $\mathcal{M}-\mathcal{Y}$ transition across maps of adjacent values of $n$ is continuous (white dashed curves). Any $\mathcal{M}-\mathcal{X}$ transition must be continuous because, at the transition point, the structures involved correspond to the same affine stretch of a locally densest structure at $D=D_{\min }$ along the $x$ direction. As illustrated in Fig. 7, the discontinuity of any $\mathcal{X}-\mathcal{Y}$ transition is due to the predefined difference in stretch direction between a type- $\mathcal{X}$ and a type- $\mathcal{Y}$ structure. For any cross-map $\mathcal{M}-\mathcal{Y}$ transition, the type- $\mathcal{M}$ structure at $D=D_{\min }$ of the $(n+1)$ regime corresponds to an affine stretch along the $y$ direction, and is therefore identical to the type- $\mathcal{Y}$ structure of the $n$ regime. For any transition, the packing fraction $\phi$ is continuous but nonsmooth. This is illustrated by the upper bound of each blue region in Fig. 6.

[1] P. M. Chaikin and T. C. Lubensky, Principles of Condensed Matter Physics (Cambridge University, Cambridge, England 2000).

[2] A. Donev, F. H. Stillinger, P. M. Chaikin, and S. Torquato, Unusually Dense Crystal Packings of Ellipsoids, Phys. Rev. Lett. 92, 255506 (2004).

[3] J. D. Bernal, Geometry of the structure of monatomic liquids, Nature (London) 185, 68 (1960).

[4] J. L. Finney, Bernal's road to random packing and the structure of liquids, Philos. Mag. 93, 3940 (2013).
For any given value of $n$, all type- $\mathcal{M}$ structures share the same packing fraction given by Eq. (3) regardless of their values of $D$ and $A$, because they are all affine transformations of the same locally densest structure of circular disks. The packing fraction of any type- $\mathcal{M}$ structure is therefore only determined by the corresponding value of $n$. Any cross-map $\mathcal{M}-\mathcal{M}$ transition across adjacent values of $n$ is not possible, because the packing fraction of a type- $\mathcal{M}$ structure at $n$ must be different from that of a type- $\mathcal{M}$ structure at $n+1$. The above-mentioned requirement for $\phi$ to be continuous in a structural transition cannot be fulfilled. Such discontinuity in $\phi$ between type- $\mathcal{M}$ structures of adjacent values of $n$ is illustrated in Fig. 6 by the corresponding upper bounds for densest packings, and in Fig. 8(b) by the color contrast between the type- $\mathcal{M}$ regions of $n=2$ and 3 .

\section{CONCLUSIONS AND OUTLOOK}

The findings presented in this paper enrich the field of condensed matter physics with a class of low-dimensional, orientationally ordered crystal structures, and reveal an intriguing connection of such crystal structures of ellipses with known densest-packed structures of circular disks. Our analytic theory, which agrees perfectly with the corresponding results from Monte Carlo simulations, provides a unified explanation of how this class of crystals emerges as candidates for densest-packed structures. It also offers possibilities of developing materials or devices with specific orientational ordering. Such confinement-induced crystal structures of ellipses may be used in the development of phononic or photonic materials with the aim to tailor classical-wave properties. For example, they may serve as building blocks for acoustic devices formed by granular crystals [72-74] and for self-assembled colloidal photonic crystals [75-77]. Future work should include a study of the phase diagrams [57-59,61,62] of such confined systems, as well as an extension of this research to binary mixtures of ellipses [78,79] and to driven granular systems of elliptical particles under cyclic shear by a parallel pair of moving boundaries [80,81].

\section{ACKNOWLEDGMENTS}

This work was supported by the National Natural Science Foundation of China (Grant No. 11932005), as well as the Science, Technology, and Innovation Commission of Shenzhen Municipality (Grants No. JCYJ20160531193515801 and No. ED11409002).
[5] G. Parisi and F. Zamponi, Mean-field theory of hard sphere glasses and jamming, Rev. Mod. Phys. 82, 789 (2010).

[6] S. Torquato, Random Heterogeneous Materials: Microstructure and Macroscopic Properties (Springer, New York, 2002).

[7] H. Loewen, Twenty years of confined colloids: From confinement-induced freezing to giant breathing, J. Phys.: Condens. Matter 21, 474203 (2009).

[8] Z. Zheng, F. Wang, and Y. Han, Glass Transitions in Quasi-TwoDimensional Suspensions of Colloidal Ellipsoids, Phys. Rev. Lett. 107, 065702 (2011). 
[9] U. Tkalec and I. Muševič, Topology of nematic liquid crystal colloids confined to two dimensions, Soft Matter 9, 8140 (2013).

[10] Z. Zheng, R. Ni, F. Wang, M. Dijkstra, Y. Wang, and Y. Han, Structural signatures of dynamic heterogeneities in monolayers of colloidal ellipsoids, Nat. Commun. 5, 3829 (2014).

[11] S. Ramananarivo, E. Ducrot, and J. Palacci, Activity-controlled annealing of colloidal monolayers, Nat. Commun. 10, 3380 (2019).

[12] X. Liang, R. Dong, and J. C. Ho, Self-assembly of colloidal spheres toward fabrication of hierarchical and periodic nanostructures for technological applications, Adv. Mater. Technol. 4, 1800541 (2019)

[13] K. Zhao, R. Bruinsma, and T. G. Mason, Entropic crystalcrystal transitions of Brownian squares, Proc. Natl. Acad. Sci. USA 108, 2684 (2011).

[14] T. Paik and C. B. Murray, Shape-directed binary assembly of anisotropic nanoplates: A nanocrystal puzzle with shapecomplementary building blocks, Nano Lett. 13, 2952 (2013).

[15] J. A. Millan, D. Ortiz, and S. C. Glotzer, Effect of shape on the self-assembly of faceted patchy nanoplates with irregular shape into tiling patterns, Soft Matter 11, 1386 (2015).

[16] J.-W. Kim, S. J. Lee, P. Biswas, T. I. Lee, and J.-M. Myoung, Solution-processed n- $\mathrm{ZnO}$ nanorod/p- $\mathrm{Co}_{3} \mathrm{O}_{4}$ nanoplate heterojunction light-emitting diode, Appl. Surf. Sci. 406, 192 (2017).

[17] Y. Zhang, B. Huang, G. Luo, T. Sun, Y. Feng, Y. Wang, Y. Ma, Q. Shao, Y. Li, Z. Zhou, and X. Huang, Atomically deviated Pd-Te nanoplates boost methanol-tolerant fuel cells, Sci. Adv. 6, eaba9731 (2020).

[18] W. Qi, J. de Graaf, F. Qiao, S. Marras, L. Manna, and M. Dijkstra, Ordered two-dimensional superstructures of colloidal octapod-shaped nanocrystals on flat substrates, Nano Lett. 12, 5299 (2012).

[19] W. Qi, J. de Graaf, F. Qiao, S. Marras, L. Manna, and M. Dijkstra, Phase diagram of octapod-shaped nanocrystals in a quasi-two-dimensional planar geometry, J. Chem. Phys. 138, 154504 (2013).

[20] M. P. Arciniegas, M. R. Kim, J. De Graaf, R. Brescia, S. Marras, K. Miszta, M. Dijkstra, R. van Roij, and L. Manna, Self-assembly of octapod-shaped colloidal nanocrystals into a hexagonal ballerina network embedded in a thin polymer film, Nano Lett. 14, 1056 (2014).

[21] M. Treier, P. Ruffieux, R. Fasel, F. Nolting, S. Yang, L. Dunsch, and T. Greber, Looking inside an endohedral fullerene: Interand intramolecular ordering of $\mathrm{Dy}_{3} \mathrm{~N} @ \mathrm{C}_{80}\left(I_{h}\right)$ on $\mathrm{Cu}(111)$, Phys. Rev. B 80, 081403(R) (2009).

[22] W. Mickelson, S. Aloni, W.-Q. Han, J. Cumings, and A. Zettl, Packing $\mathrm{C}_{60}$ in Boron Nitride Nanotubes, Science 300, 467 (2003).

[23] A. N. Khlobystov, D. A. Britz, A. Ardavan, and G. A. D. Briggs, Observation of Ordered Phases of Fullerenes in Carbon Nanotubes, Phys. Rev. Lett. 92, 245507 (2004).

[24] V. T. Cong, E.-O. Ganbold, J. K. Saha, J. Jang, J. Min, J. Choo, S. Kim, N. W. Song, S. J. Son, S. B. Lee, and S.-W. Joo, Gold nanoparticle silica nanopeapods, J. Am. Chem. Soc. 136, 3833 (2014).

[25] R. Liang, J. Xu, R. Deng, K. Wang, S. Liu, J. Li, and J. Zhu, Assembly of polymer-tethered gold nanoparticles under cylindrical confinement, ACS Macro Lett. 3, 486 (2014).
[26] S. Sanwaria, A. Horechyy, D. Wolf, C.-Y. Chu, H.-L. Chen, P. Formanek, M. Stamm, R. Srivastava, and B. Nandan, Helical packing of nanoparticles confined in cylindrical domains of a self-assembled block copolymer structure, Angew. Chem. Int Ed. 53, 9090 (2014).

[27] Z. Chen, S. Ye, S. D. Evans, Y. Ge, Z. Zhu, Y. Tu, and X. Yang, Confined assembly of hollow carbon spheres in carbonaceous nanotube: A spheres-in-tube carbon nanostructure with hierarchical porosity for high-performance supercapacitor, Small 14, 1704015 (2018).

[28] J. H. Moon, S. Kim, G.-R. Yi, Y.-H. Lee, and S.-M. Yang, Fabrication of ordered macroporous cylinders by colloidal templating in microcapillaries, Langmuir 20, 2033 (2004).

[29] F. Li, X. Badel, J. Linnros, and J. B. Wiley, Fabrication of colloidal crystals with tubular-like packings, J. Am. Chem. Soc. 127, 3268 (2005).

[30] M. Tymczenko, L. F. Marsal, T. Trifonov, I. Rodriguez, F. Ramiro-Manzano, J. Pallares, A. Rodriguez, R. Alcubilla, and F. Meseguer, Colloidal crystal wires, Adv. Mater. 20, 2315 (2008).

[31] S. A. Vanapalli, C. R. Iacovella, K. E. Sung, D. Mukhija, J. M. Millunchick, M. A. Burns, S. C. Glotzer, and M. J. Solomon, Fluidic assembly and packing of microspheres in confined channels, Langmuir 24, 3661 (2008).

[32] A. J. Meagher, F. García-Moreno, J. Banhart, A. Mughal, and S. Hutzler, An experimental study of columnar crystals using monodisperse microbubbles, Colloids Surf. A 473, 55 (2015).

[33] T. Lee, K. Gizynski, and B. A. Grzybowski, Non-equilibrium self-assembly of monocomponent and multicomponent tubular structures in rotating fluids, Adv. Mater. 29, 1704274 (2017).

[34] G. T. Pickett, M. Gross, and H. Okuyama, Spontaneous Chirality in Simple Systems, Phys. Rev. Lett. 85, 3652 (2000).

[35] A. Mughal, H.-K. Chan, and D. Weaire, Phyllotactic Description of Hard Sphere Packing in Cylindrical Channels, Phys. Rev. Lett. 106, 115704 (2011).

[36] H.-K. Chan, Densest columnar structures of hard spheres from sequential deposition, Phys. Rev. E 84, 050302(R) (2011).

[37] A. Mughal, H.-K. Chan, D. Weaire, and S. Hutzler, Dense packings of spheres in cylinders: Simulations, Phys. Rev. E 85 , 051305 (2012).

[38] H.-K. Chan, A hybrid helical structure of hard-sphere packing from sequential deposition, Philos. Mag. 93, 4057 (2013).

[39] A. Mughal, Screw symmetry in columnar crystals, Philos. Mag. 93, 4070 (2013).

[40] A. Mughal and D. Weaire, Theory of cylindrical dense packings of disks, Phys. Rev. E 89, 042307 (2014).

[41] M. Z. Yamchi and R. K. Bowles, Helical Defect Packings in a Quasi-One-Dimensional System of Cylindrically Confined Hard Spheres, Phys. Rev. Lett. 115, 025702 (2015).

[42] L. Fu, W. Steinhardt, H. Zhao, J. E. S. Socolarb, and P. Charbonneau, Hard sphere packings within cylinders, Soft Matter 12, 2505 (2016).

[43] L. Fu, C. Bian, C. Wyatt Shields IV, D. F. Cruz, G. P. López, and P. Charbonneau, Assembly of hard spheres in a cylinder: A computational and experimental study, Soft Matter 13, 3296 (2017).

[44] A. Mughal, J. Winkelmann, D. Weaire, and S. Hutzler, Columnar structures of soft spheres: Metastability and hysteresis, Phys. Rev. E 98, 043303 (2018). 
[45] J. Winkelmann, B. Haffner, D. Weaire, A. Mughal, and S. Hutzler, Corrected Article: Simulation and observation of line-slip structures in columnar structures of soft spheres [Phys. Rev. E 96, 012610 (2017)], Phys. Rev. E 97, 059902 (2018).

[46] J. Winkelmann, A. Mughal, D. B. Williams, D. Weaire, and S. Hutzler, Theory of rotational columnar structures of soft spheres, Phys. Rev. E 99, 020602(R) (2019).

[47] J. Winkelmann, A. Mughal, D. Weaire, and S. Hutzler, Equilibrium configurations of hard spheres in a cylindrical harmonic potential, Europhys. Lett. 127, 44002 (2019).

[48] H.-K. Chan, Y. Wang, and H. Han, Densest helical structures of hard spheres in narrow confinement: An analytic derivation, AIP Adv. 9, 125118 (2019).

[49] W. Jin, Y. Jiao, L. Liu, Y. Yuan, and S. Li, Dense crystalline packings of ellipsoids, Phys. Rev. E 95, 033003 (2017).

[50] S. Torquato and Y. Jiao, Dense packings of the Platonic and Archimedean solids, Nature (London) 460, 876 (2009).

[51] E. R. Chen, D. Klotsa, M. Engel, P. F. Damasceno, and S. C. Glotzer, Complexity in Surfaces of Densest Packings for Families of Polyhedra, Phys. Rev. X 4, 011024 (2014).

[52] R. Ni, A. P. Gantapara, J. de Graaf, R. van Roij, and M. Dijkstra, Phase diagram of colloidal hard superballs: From cubes via spheres to octahedra, Soft Matter 8, 8826 (2012).

[53] W. Jin, P. Lu, and S. Li, Evolution of the dense packings of spherotetrahedral particles: From ideal tetrahedra to spheres, Sci. Rep. 5, 15640 (2015).

[54] W. Jin, H.-K. Chan, and Z. Zhong, Shape-Anisotropy-Induced Ordered Packings in Cylindrical Confinement, Phys. Rev. Lett. 124, 248002 (2020).

[55] Z. Füredi, The densest packing of equal circles into a parallel strip, Discrete Comput. Geom. 6, 95 (1991)

[56] T. Matsumoto and W. Nowacki, On densest packings of ellipsoids, Z. Kristallogr. Cryst. Mater. 123, 401 (1966).

[57] J. Vieillard-Baron, Phase transitions of the classical hard-ellipse system, J. Chem. Phys. 56, 4729 (1972).

[58] J. A. Cuesta and D. Frenkel, Monte Carlo simulation of twodimensional hard ellipses, Phys. Rev. A 42, 2126 (1990).

[59] M. A. Bates and D. Frenkel, Phase behavior of two-dimensional hard rod fluids, J. Chem. Phys. 112, 10034 (2000).

[60] A. Donev, R. Connelly, F. H. Stillinger, and S. Torquato, Underconstrained jammed packings of nonspherical hard particles: Ellipses and ellipsoids, Phys. Rev. E 75, 051304 (2007).

[61] W.-S. Xu, Y.-W. Li, Z.-Y. Sun, and L.-J. An, Hard ellipses: Equation of state, structure, and self-diffusion, J. Chem. Phys. 139, 024501 (2013).

[62] G. Bautista-Carbajal and G. Odriozola, Phase diagram of twodimensional hard ellipses, J. Chem. Phys. 140, 204502 (2014).

[63] J. Kallrath and S. Rebennack, Cutting ellipses from area-minimizing rectangles, J. Glob. Optim. 59, 405 (2014).

[64] D. N. Ilin and M. Bernacki, Advancing layer algorithm of dense ellipse packing for generating statistically equivalent polygonal structures, Granul. Matter 18, 43 (2016).

[65] D. N. Ilin and M. Bernacki, A new algorithm for dense ellipse packing and polygonal structures generation in context of FEM or DEM, MATEC Web Conf. 80, 02004 (2016).

[66] A. Pankratov, T. Romanova, and I. Litvinchev, Packing ellipses in an optimized rectangular container, Wireless Netw. 26, 4869 (2020).

[67] K. Xu, Geometric formulas of Lewis's law and Aboav-Weaire's law in two dimensions based on ellipse packing, Philos. Mag. Lett. 99, 317 (2019).

[68] G. Delaney, D. Weaire, S. Hutzler, and S. Murphy, Random packing of elliptical disks, Philos. Mag. Lett. 85, 89 (2005).

[69] H. B. Aaron and G. F. Bolling, Free volume as a criterion for grain boundary models, Surf. Sci. 31, 27 (1972).

[70] M. Tanemura and T. Matsumoto, Density of the $p 2 g g-4 \mathrm{c} 1$ packing of ellipses (II), Z. Kristallogr. Cryst. Mater. 230, 709 (2015).

[71] D. Frenkel and R. Eppenga, Evidence for algebraic orientational order in a two-dimensional hard-core nematic, Phys. Rev. A 31, 1776 (1985).

[72] D. Ngo, D. Khatri, and C. Daraio, Highly nonlinear solitary waves in chains of ellipsoidal particles, Phys. Rev. E 84, 026610 (2011).

[73] J. Lydon, M. Serra-Garcia, and C. Daraio, Local to Extended Transitions of Resonant Defect Modes, Phys. Rev. Lett. 113, 185503 (2014).

[74] A. Merkel and J. Christensen, Ultrasonic nodal chains in topological granular metamaterials, Commun. Phys. 2, 1 (2019).

[75] Q.-L. Lei, R. Ni, and Y.-Q. Ma, Self-assembled chiral photonic crystals from a colloidal helix racemate, ACS Nano 12, 6860 (2018).

[76] R. K. Cersonsky, J. Dshemuchadse, J. Antonaglia, G. van Anders, and S. C. Glotzer, Pressure-tunable photonic band gaps in an entropic colloidal crystal, Phys. Rev. Mater. 2, 125201 (2018).

[77] D. Wan and S. C. Glotzer, Randomness in self-assembled colloidal crystals can widen photonic band gaps through particle shape and internal structure, arXiv:2010.04983 (2020).

[78] C. E. Zachary, Y. Jiao, and S. Torquato, Hyperuniformity, quasi-long-range correlations, and void-space constraints in maximally random jammed particle packings. II. Anisotropy in particle shape, Phys. Rev. E 83, 051309 (2011).

[79] W.-S. Xu, Z.-Y. Sun, and L.-J. An, Relaxation dynamics in a binary hard-ellipse liquid, Soft Matter 11, 627 (2015).

[80] M. Nicolas, P. Duru, and O. Pouliquen, Compaction of a granular material under cyclic shear, Eur. Phys. J. E 3, 309 (2000).

[81] W. Jin, C. S. O'Hern, C. Radin, M. D. Shattuck, and H. L. Swinney, Homogeneous Crystallization in Cyclically Sheared Frictionless Grains, Phys. Rev. Lett. 125, 258003 (2020). 